\title{
Prime divisors of Lucas sequences
}

\author{
by \\ Pieter Moree (Bonn) and Peter Stevenhagen (Amsterdam)
}

1. Introduction. Let $d>1$ be a squarefree integer and $K=\mathbb{Q}(\sqrt{d})$ the corresponding real quadratic field. We write $\varepsilon=a+b \sqrt{d}$ for a fundamental unit in the ring of integers of $K$, and $\bar{\varepsilon}$ for its conjugate. The Lucas sequence associated with $K$ is the integer sequence

$$
X_{K}=\left\{\operatorname{Tr}_{K / \mathbb{Q}}\left(\varepsilon^{n}\right)\right\}_{n=0}^{\infty}=\left\{\varepsilon^{n}+\bar{\varepsilon}^{n}\right\}_{n=0}^{\infty} .
$$

For odd $n$ the sign of $x_{n}$ depends on the choice of the sign of $a$. This is irrelevant for the divisibility properties we will be concerned with, but for uniqueness sake we take $x_{1}=2 a>0$.

The Lucas sequence $X_{K}$ satisfies the second order linear recurrence

$$
x_{n+2}=2 a x_{n+1}-N_{K / \mathbb{Q}}(\varepsilon) x_{n}
$$

for $n \geq 0$. If we take for $K$ the field $\mathbb{Q}(\sqrt{5})$ generated by the golden ratio, we obtain the very classical example of the Lucas sequence defined by the "Fibonacci recursion" $x_{n+2}=x_{n+1}+x_{n}$ with initial values $x_{0}=2$ and $x_{1}=1$.

In this note, we show that the set of prime numbers $p$ that divide some term of the sequence $X_{K}$ has a natural density $\delta_{K}$ and determine it for each $K$. More precisely, we compute the density $\delta_{K}^{+}$of the primes that split completely in $K$ and divide some term of $X_{K}$ and the density $\delta_{K}^{-}$of the primes that are inert in $K$ and divide some term of $X_{K}$. The arguments for both kinds of primes are somewhat different, and so are the associated densities. It turns out that the determination of $\delta_{K}^{-}$is the more difficult part, unless we are in the "easy case" in which the norm $N_{K / \mathbb{Q}}(\varepsilon)$ equals -1 , when it is trivially determined. Clearly, one has $\delta_{K}=\delta_{K}^{+}+\delta_{K}^{-}$.

The method in this note extends to sequences $\left\{\operatorname{Tr}_{K / \mathbb{Q}}\left(\alpha^{n}\right)\right\}_{n=0}^{\infty}=\left\{\alpha^{n}+\right.$ $\left.\bar{\alpha}^{n}\right\}_{n=0}^{\infty}$, where $\alpha$ is any algebraic integer in a quadratic field $K$. Although it is a bit cumbersome to express the density as an explicit rational number

1991 Mathematics Subject Classification: Primary 11R45; Secondary 11B39.

Key words and phrases: Lucas sequence, Chebotarev density theorem. 
in terms of $\alpha$, this yields a proof of what is called a "main conjecture" in [5, p. 362]. For more details, we refer to the treatment of general second order "torsion sequences" in [6].

Theorem. Let $K=\mathbb{Q}(\sqrt{d})$ and $a=\frac{1}{2} \operatorname{Tr}_{K / \mathbb{Q}}(\varepsilon)>0$ be as above. Then the natural densities $\delta_{K}^{+}$and $\delta_{K}^{-}$for the sets of prime divisors of the Lucas sequence associated with $K$ exist. For $N_{K / \mathbb{Q}}(\varepsilon)=-1$ the densities are as follows.

\begin{tabular}{ccr}
\hline & $d=2$ & $d>2$ \\
\hline$\delta_{K}^{+}$ & $11 / 24$ & $5 / 12$ \\
$\delta_{K}^{-}$ & $1 / 4$ & $1 / 4$ \\
$\delta_{K}$ & $17 / 24$ & $2 / 3$ \\
\hline
\end{tabular}

For $N_{K / \mathbb{Q}}(\varepsilon)=1$ the densities depend in the following way on whether $a+1$ and $a-1$ are rational squares or not.

\begin{tabular}{cccc}
\hline & $a-1=\square$ & $a+1=\square$ & $a \pm 1 \neq \square$ \\
\hline$\delta_{K}^{+}$ & $5 / 24$ & $5 / 24$ & $1 / 6$ \\
$\delta_{K}^{K}$ & $1 / 8$ & $5 / 24$ & $1 / 6$ \\
$\delta_{K}$ & $1 / 3$ & $5 / 12$ & $1 / 3$ \\
\hline
\end{tabular}

The main ingredient of the proof is the Chebotarev density theorem, and the basic idea of the method goes back to Hasse [2]. Lagarias [3] was the first to use this idea in a quadratic setting, for the classical Lucas sequence mentioned above, which falls in the category $N_{K / \mathbb{Q}}(\varepsilon)=-1$. A generalization to other instances of units of norm -1 is given in [4]. For the easier and well-studied case of reducible second order recurrences $\left\{r^{n}+s^{n}\right\}_{n=0}^{\infty}$ with $r, s \in \mathbb{Z}$, or for generalizations to higher order linear recurrences, the reader can consult [1].

2. Proof of the Theorem. Let $K=\mathbb{Q}(\sqrt{d})$ and $\varepsilon=a+b \sqrt{d}$ be as before, and write $\mathcal{O}$ for the ring of integers of $K$. If $p$ is a prime that is unramified in $K / \mathbb{Q}$, then the kernel of the norm map $\kappa_{p}=\operatorname{ker}\left[N:(\mathcal{O} / p \mathcal{O})^{*} \rightarrow \mathbb{F}_{p}^{*}\right]$ is a cyclic group of order $p-\left(\frac{d}{p}\right)$. We set

$$
q=q_{K}=\varepsilon / \bar{\varepsilon}= \begin{cases}-\varepsilon^{2} & \text { if } N_{K / \mathbb{Q}}(\varepsilon)=-1, \\ \varepsilon^{2} & \text { if } N_{K / \mathbb{Q}}(\varepsilon)=1 .\end{cases}
$$

Let $p \nmid 2 d$ be a prime number. Looking at the explicit form of the $n$th term $x_{n}=\varepsilon^{n}+\bar{\varepsilon}^{n}$ of $X_{K}$, we find that $p$ divides $x_{n}$ if and only if we have $q^{n}=-1 \in(\mathcal{O} / p \mathcal{O})^{*}$. As $q$ lies in the cyclic subgroup $\kappa_{p} \subset(\mathcal{O} / p \mathcal{O})^{*}$ and -1 is the unique element of order 2 in that group, we find the basic characterization

$p$ divides some term of $X_{K} \Leftrightarrow$ the order of $q \in(\mathcal{O} / p \mathcal{O})^{*}$ is even. 
The key idea in determining the densities $\delta_{K}^{+}$and $\delta_{K}^{-}$is that one can describe the parity of the order of $q \in(\mathcal{O} / p \mathcal{O})^{*}$ in terms of the splitting behavior of $p$ in some infinite algebraic extension of $\mathbb{Q}$. We start with the easier case of the rational primes that split completely in $K$.

Split case. Let $S^{+}$be the set of odd primes $p$ that split completely in $K$, and $D^{+} \subset S^{+}$the set of primes in $S^{+}$that divide some term of $X_{K}$.

For $k \in \mathbb{Z}_{\geq 1}$, we let $S_{k}^{+} \subset S^{+}$be the set of primes $p \in S^{+}$for which $p-1$ has exactly $k=\operatorname{ord}_{2}(p-1)$ factors 2 . The set $S_{k}^{+}$consists of the primes that split completely in the field $K\left(\zeta_{2^{k}}\right)$ obtained by adjoining to $K$ a primitive $2^{k}$ th root of unity, but not in the field $K\left(\zeta_{2^{k+1}}\right)$ obtained by adjoining to $K$ a primitive $2^{k+1}$ th root of unity. By the Chebotarev density theorem, the set $S_{k}^{+}$has a natural density inside the set of all primes. It equals $\delta\left(S_{k}^{+}\right)=\left[K\left(\zeta_{2^{k}}\right): \mathbb{Q}\right]^{-1}-\left[K\left(\zeta_{2^{k+1}}\right): \mathbb{Q}\right]^{-1}$. Clearly, the sum of these densities for all $k \geq 1$ is $[K: \mathbb{Q}]^{-1}=1 / 2=\delta\left(S^{+}\right)$.

For $p \in S_{k}^{+}$, the group $(\mathcal{O} / p \mathcal{O})^{*}$ is a product of two cyclic groups of order $p-1$, and an element has odd order in $(\mathcal{O} / p \mathcal{O})^{*}$ if and only if it is a $2^{k}$ th power in $(\mathcal{O} / p \mathcal{O})^{*}$. As $q \in(\mathcal{O} / p \mathcal{O})^{*}$ is a $2^{k}$ th power if and only if $p$ splits completely in the field $K\left(\zeta_{2^{k}}, \sqrt[2^{k}]{q}\right)$, we conclude that a prime $p \in S_{k}^{+}$does not divide a term of $X_{K}$ if and only if it splits completely in $K\left(\zeta_{2^{k}}, \sqrt[2 k]{q}\right)$, but not in $K\left(\zeta_{2^{k+1}}, \sqrt[2^{k}]{q}\right)$. By the Chebotarev density theorem, the subset of such primes in $S_{k}^{+}$has natural density $\left[K\left(\zeta_{2^{k}}, \sqrt[2 k]{q}\right): \mathbb{Q}\right]^{-1}-\left[K\left(\zeta_{2^{k+1}}, \sqrt[2^{k}]{q}\right): \mathbb{Q}\right]^{-1}$. The complement $D_{k}^{+}=D^{+} \cap S_{k}^{+}$of this set in $S_{k}^{+}$has a density as well, and we find that both $D^{+}=\bigcup_{k \geq 1} D_{k}^{+}$and its complement $S^{+} \backslash D^{+}=$ $\bigcup_{k \geq 1}\left(S_{k}^{+} \backslash D_{k}^{+}\right)$in $S^{+}$are countable disjoint unions of sets of primes having a natural density. It follows that $D^{+}$has lower density $\sum_{k \geq 1} \delta\left(D_{k}^{+}\right)$, and that $S^{+} \backslash D^{+}$has lower density $\sum_{k>1} \delta\left(S_{k}^{+} \backslash D_{k}^{+}\right)$. These lower densities add up to $\delta\left(S^{+}\right)$, so they are in fact densities. We conclude that $D^{+}$has a natural density $\delta_{K}^{+}$which satisfies

$$
\frac{1}{2}-\delta_{K}^{+}=\sum_{k \geq 1}\left(\frac{1}{\left[K\left(\zeta_{2^{k}}, \sqrt[2^{k}]{q}\right): \mathbb{Q}\right]}-\frac{1}{\left[K\left(\zeta_{2^{k+1}}, \sqrt[2^{k}]{q}\right): \mathbb{Q}\right]}\right) .
$$

Equation (2.2) reduces the computation of $\delta_{K}^{+}$to a computation of field degrees in the infinite extension $K\left(\zeta_{2}, \sqrt[2 \infty]{q}\right)$ of $\mathbb{Q}$. To ease notation, we write

$$
F_{k}=K\left(\zeta_{2^{k+1}}, \sqrt[2^{k}]{q}\right)
$$

Then the $k$ th term of the right hand side of $(2.2)$ equals $\left[F_{k}: \mathbb{Q}\right]^{-1}$ if $\zeta_{2^{k+1}}$ generates a quadratic extension of $K\left(\zeta_{2^{k}}, \sqrt[2 k]{q}\right)$, and 0 otherwise.

Suppose first that we have $N_{K / \mathbb{Q}}(\varepsilon)=-1$, and consequently $q=-\varepsilon^{2}$ in (2.1). Then $q$ is a square in $K\left(\zeta_{4}\right)$, and also in the field $M=K\left(\zeta_{2} \infty\right)$ obtained by adjoining all 2 -power roots of unity to $K$. It is not a fourth 
power in $M$, since $M$ is abelian over $\mathbb{Q}$ and $M(\sqrt[4]{q})=M(\sqrt{\varepsilon})$ has a quartic subfield $K(\sqrt{\varepsilon})$ that is not normal over $\mathbb{Q}$. By Kummer theory, it follows that $\sqrt[2^{k}]{q}$ generates a cyclic extension of degree $2^{k-1}$ of $K\left(\zeta_{2^{k}}\right)$ for every $k \geq 2$. Our normality argument shows that this extension is linearly disjoint over $K\left(\zeta_{2^{k}}\right)$ from $K\left(\zeta_{2^{k+1}}\right)$.

For $k=1$, we have a quadratic extension $K(\sqrt{q})=K\left(\zeta_{4}\right)$, which coincides with the extension generated by $\zeta_{2^{k+1}}=\zeta_{4}$. This shows that in the case of norm -1 , the term for $k=1$ in $(2.2)$ vanishes.

If $K$ is not the real quadratic subfield $\mathbb{Q}(\sqrt{2})$ of $\mathbb{Q}\left(\zeta_{2^{\infty}}\right)$, then $\zeta_{2^{k+1}}$ generates a quadratic extension of $K\left(\zeta_{2^{k}}\right)$ for all $k \geq 2$, and $F_{k}$ has degree $2 \cdot 2^{k} \cdot 2^{k-1}=4^{k}$ for these $k$. We find $1 / 2-\delta_{K}^{+}=\sum_{k \geq 2} 4^{-k}=1 / 12$ and $\delta_{K}^{+}=5 / 12$.

For $K=\mathbb{Q}(\sqrt{2})$ the degree of $F_{k}$ is only $2^{k} \cdot 2^{k-1}=2^{2 k-1}$ for $k \geq 3$. Moreover, the term for $k=2$ in $(2.2)$ vanishes since $K\left(\zeta_{4}\right)=\mathbb{Q}\left(\zeta_{8}\right)$ now contains $\zeta_{2^{k+1}}=\zeta_{8}$. We find $1 / 2-\delta_{K}^{+}=\sum_{k \geq 3} 2^{1-2 k}=1 / 24$ and $\delta_{K}^{+}=$ $11 / 24$.

The diagrams below indicate the field degrees in the two situations for $k \geq 2$ and $k \geq 3$, respectively.
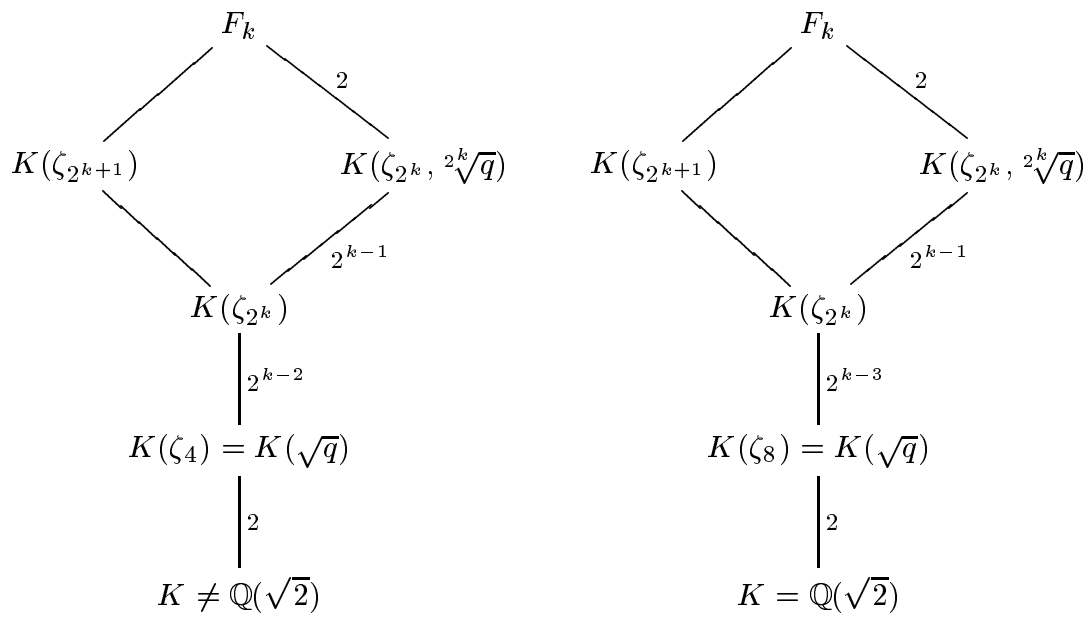

Suppose next that we have $N_{K / \mathbb{Q}}(\varepsilon)=1$, and so in particular $K \neq$ $\mathbb{Q}(\sqrt{2})$. The analysis is similar to the previous case, but we now have $q=\varepsilon^{2}$, so $q$ is a square in $K$. As the field $K(\sqrt[4]{\varepsilon})$ is non-normal of degree 8 , we see that $q=\varepsilon^{2}$ is a square in $M=K\left(\zeta_{2} \infty\right)$, but not an eighth power. We have two cases.

Suppose $q$ is not a fourth power in $M$. Then $\sqrt[2 k]{q}$ generates a cyclic extension of degree $2^{k-1}$ of $K\left(\zeta_{2^{k}}\right)$ for every $k \geq 1$, and this extension is linearly disjoint from the extension of $K\left(\zeta_{2^{k}}\right)$ generated by $\zeta_{2^{k+1}}$. This is similar to the case $K \neq \mathbb{Q}(\sqrt{2})$ above, the only difference being that we 
now also have a non-zero term for $k=1$ in $(2.2)$. We find $1 / 2-\delta_{K}^{+}=$ $\sum_{k \geq 1} 4^{-k}=1 / 3$ and $\delta_{K}^{+}=1 / 6$.

Suppose that $q$ is a fourth power in $M$. Then $K(\sqrt{\varepsilon})$ is a subfield of $M$. Besides $K$, the quadratic subfields of $K(\sqrt{\varepsilon})$ are the two fields $\mathbb{Q}(\sqrt{\varepsilon} \pm 1 / \sqrt{\varepsilon})$, and one of those two is contained in $\mathbb{Q}\left(\zeta_{2} \infty\right)$. From $N_{K / \mathbb{Q}}(\varepsilon)=1$ we deduce

$$
(\sqrt{\varepsilon} \pm 1 / \sqrt{\varepsilon})^{2}=\operatorname{Tr}_{K / \mathbb{Q}}(\varepsilon) \pm 2=2(a \pm 1),
$$

so this "exceptional case" occurs exactly when one of the elements $a \pm 1$ is a rational square. The fields $K\left(\zeta_{4}, \sqrt[4]{q}\right)=K\left(\zeta_{4}, \sqrt{\varepsilon}\right)$ and $K\left(\zeta_{8}\right)$ coincide here, and $\sqrt[2^{k}]{q}$ generates, for all $k \geq 3$, a cyclic extension of degree $2^{k-2}$ of $K\left(\zeta_{2^{k}}\right)$ that is linearly disjoint over $K\left(\zeta_{2^{k}}\right)$ from $K\left(\zeta_{2^{k+1}}\right)$. As in the case $K=\mathbb{Q}(\sqrt{2})$ above, the degree of $F_{k}$ is only $2^{k} \cdot 2^{k-1}=2^{2 k-1}$ for $k \geq 3$. The term for $k=2$ vanishes, but for $k=1$ we do have a contribution $1 / 4$. We find $1 / 2-\delta_{K}^{+}=1 / 4+\sum_{k \geq 3} 2^{1-2 k}=7 / 24$ and $\delta_{K}^{+}=5 / 24$ if either $a+1$ or $a-1$ is a square. This shows that the values of $\delta_{K}^{+}$are as asserted.

Inert case. Let $p$ be a prime that is inert in $K / \mathbb{Q}$. Then $\mathcal{O} / p \mathcal{O}$ is a field of $p^{2}$ elements, and the norm map $N: \mathcal{O} / p \mathcal{O} \rightarrow \mathbb{F}_{p}$ raises all elements to the power $p+1$.

Suppose first that we are in the case $N_{K / \mathbb{Q}}(\varepsilon)=-1$. Then we have $q=-\varepsilon^{2}$ in $(2.1)$ and $\varepsilon^{p+1}=-1 \in(\mathcal{O} / p \mathcal{O})^{*}$. For $p \equiv 1 \bmod 4$ we obtain $q^{(p+1) / 2}=-\varepsilon^{p+1}=1 \in(\mathcal{O} / p \mathcal{O})^{*}$, which shows that the order of $q$ in $(\mathcal{O} / p \mathcal{O})^{*}$ is odd. For $p \equiv 3 \bmod 4$ we obtain $q^{(p+1) / 2}=\varepsilon^{p+1}=-1 \epsilon$ $(\mathcal{O} / p \mathcal{O})^{*}$, which shows that the order of $q$ in $(\mathcal{O} / p \mathcal{O})^{*}$ is even. We find that $\delta_{K}^{-}$is the density of the primes $p \equiv 3 \bmod 4$ that are inert in $K / \mathbb{Q}$, hence $\delta_{K}^{-}=1 / 4$.

From now on we suppose $N_{K / \mathbb{Q}}(\varepsilon)=1$. In particular, this implies $K \neq$ $\mathbb{Q}(\sqrt{2})$. We have $q=\varepsilon^{2}$, and consequently $q^{(p+1) / 2}=\varepsilon^{p+1}=1 \in(\mathcal{O} / p \mathcal{O})^{*}$ for all inert odd primes $p$. This shows that for all inert primes $p \equiv 1 \bmod 4$, the order of $q$ is again odd and $p$ does not divide a term of $X_{K}$. For the inert primes $p \equiv 3 \bmod 4$ we use an approach that is similar to that in the split case.

Let $S^{-}$be the set of odd primes $p$ that are inert in $K / \mathbb{Q}$, and $D^{-} \subset S^{-}$ the set of primes in $S^{-}$that divide some term of $X_{K}$. For $k \in \mathbb{Z}_{\geq 2}$, we let $S_{k}^{-} \subset S^{-}$be the set of primes $p \in S^{-}$for which $p+1$ has exactly $k=\operatorname{ord}_{2}(p+1)$ factors 2 . This is a set with a natural density, and we want to compute the density of the subset $D_{k}^{-}=D^{-} \cap S_{k}^{-}$by characterizing the primes $p \in D_{k}^{-}$in terms of splitting conditions on $p$ in some finite Galois extension $F_{k} / \mathbb{Q}$.

A prime $p$ is in $S_{k}^{-}$if and only if its Frobenius substitution in the abelian $\operatorname{group} \operatorname{Gal}\left(K\left(\zeta_{2^{k+1}}\right) / \mathbb{Q}\right)$ is the unique element $\varphi$ that is non-trivial on $K$ and 
acts on the $2^{k+1}$ th roots of unity as $\varphi\left(\zeta_{2^{k+1}}\right)=\zeta_{2^{k+1}}^{-1+2^{k}}$. As $K\left(\zeta_{2^{k+1}}\right)$ has degree $2^{k+1}$ over $\mathbb{Q}$, this shows that $S_{k}^{-}$has natural density $2^{-k-1}$ for all $k$. We let $B_{k} \subset K\left(\zeta_{2^{k+1}}\right)$ be the subfield corresponding to the subgroup $\langle\varphi\rangle$ of $\operatorname{Gal}\left(K\left(\zeta_{2^{k+1}}\right) / \mathbb{Q}\right)$. Note that $K\left(\zeta_{2^{k+1}}\right)=B_{k}(\varepsilon)$ is a quadratic extension of $B_{k}$.

If $p$ is in $S_{k}^{-}$, the order $p^{2}-1=(p-1)(p+1)$ of the cyclic group $(\mathcal{O} / p \mathcal{O})^{*}$ has exactly $k+1 \geq 3$ factors 2 , and $q=\varepsilon^{2} \in(\mathcal{O} / p \mathcal{O})^{*}$ has odd order if and only if $\varepsilon^{2}$ is a $2^{k+1}$ th power in $(\mathcal{O} / p \mathcal{O})^{*}$. As -1 is a $2^{k}$ th power in $(\mathcal{O} / p \mathcal{O})^{*}$, we conclude that $p \in S_{k}^{-}$does not divide any term of $X_{K}$ if and only if $\varepsilon$ is a $2^{k}$ th power in $(\mathcal{O} / p \mathcal{O})^{*}$. This leads to a characterization of the primes $p \in S_{k}^{-}$outside $D^{-}$in terms of their splitting behavior in the field

$$
F_{k}=K\left(\zeta_{2^{k+1}}, \sqrt[2^{k}]{\varepsilon}\right)=B_{k}\left(\sqrt[2^{k}]{\varepsilon}\right)
$$

they are the primes $p$ that split completely in $B_{k}$ and have extensions in $B_{k}$ that are inert in $B_{k}(\varepsilon) / B_{k}$ and split completely in $F_{k} / B_{k}(\varepsilon)$. This means that the Frobenius symbol of $p$ in the non-abelian group $\operatorname{Gal}\left(F_{k} / \mathbb{Q}\right)$, which is only determined up to conjugacy, is an element of order 2 in the normal sub$\operatorname{group} \operatorname{Gal}\left(F_{k} / B_{k}\right)$ that does not lie in the normal subgroup $\operatorname{Gal}\left(F_{k} / B_{k}(\varepsilon)\right)$. If $n_{k}$ denotes the number of such elements in $\operatorname{Gal}\left(F_{k} / \mathbb{Q}\right)$, the Chebotarev density theorem yields an inert analogue of (2.2):

$$
\frac{1}{2}-\delta_{K}^{-}=\frac{1}{4}+\sum_{k \geq 2} \frac{n_{k}}{\left[F_{k}: \mathbb{Q}\right]}=\frac{1}{4}+\sum_{k \geq 2} 2^{-k} \frac{n_{k}}{\left[F_{k}: B_{k}\right]}
$$

This time we have to do more than a degree computation, since we also need to know the structure of the group $\operatorname{Gal}\left(F_{k} / B_{k}\right)$.

Suppose first that neither $a-1$ nor $a+1$ is a square. Then the extensions $K(\sqrt{\varepsilon})$ and $K\left(\zeta_{2} \infty\right)$ are linearly disjoint over $K$, and $F_{k}$ is a cyclic extension of degree $2^{k}$ of $B_{k}(\varepsilon)=K\left(\zeta_{2^{k+1}}\right)$ generated by $\sqrt[2^{k}]{\varepsilon}$. We can extend the generator $\varphi$ of $\operatorname{Gal}\left(B_{k}(\varepsilon) / B_{k}\right)$ to an element $\varphi^{*} \in \operatorname{Gal}\left(F_{k} / B_{k}\right)$ of order 2 by setting $\varphi^{*}\left(\sqrt[2^{k}]{\varepsilon}\right)=1 / \sqrt[2^{k}]{\varepsilon}$. As $\varphi^{*}$ acts by inversion on both $\langle\varepsilon\rangle$ and $\left\langle\zeta_{2^{k}}\right\rangle$, the Galois equivariance of the Kummer pairing

$$
\operatorname{Gal}\left(F_{k} / B_{k}\left(\zeta_{2^{k+1}}\right)\right) \times\langle\varepsilon\rangle \rightarrow\left\langle\zeta_{2^{k}}\right\rangle
$$

shows that $\varphi^{*}$ commutes with all elements in $\operatorname{Gal}\left(F_{k} / B_{k}(\varepsilon)\right)$. We find

$$
\operatorname{Gal}\left(F_{k} / B_{k}\right) \cong \operatorname{Gal}\left(F_{k} / B_{k}(\varepsilon)\right) \times\left\langle\varphi^{*}\right\rangle \cong \mathbb{Z} / 2^{k} \mathbb{Z} \times \mathbb{Z} / 2 \mathbb{Z}
$$

As there are exactly two elements of order 2 in $\operatorname{Gal}\left(F_{k} / B_{k}\right)$ of the form $\left(\sigma, \varphi^{*}\right)$, this yields $n_{k}=2$ in $(2.4)$ for all $k \geq 2$. We find $1 / 2-\delta_{K}^{-}=$ $1 / 4+\sum_{k \geq 2} 2^{-k} \cdot 2 \cdot 2^{-k-1}=1 / 3$ and $\delta_{K}^{-}=1 / 6$. 


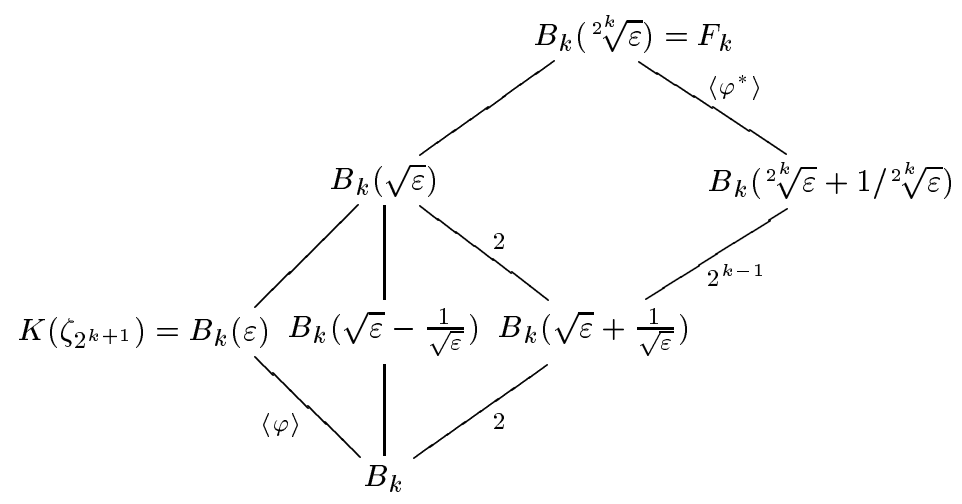

Suppose finally that we are in the exceptional case where either $a+1$ or $a-1$ is a square. Then the extension $F_{k} / B_{k}$ in the diagram above collapses to an extension of degree $2^{k}$ for all $k \geq 2$. For $k \geq 3$, we have $\sqrt{2} \in B_{k}$ and (2.3) shows that $B_{k}$ contains $\sqrt{\varepsilon}+1 / \sqrt{\varepsilon}$ if $a+1$ is a square and $\sqrt{\varepsilon}-1 / \sqrt{\varepsilon}$ if $a-1$ is a square. In the first case we have an isomorphism

$$
\operatorname{Gal}\left(F_{k} / B_{k}\right) \cong \operatorname{Gal}\left(B_{k}(\sqrt{\varepsilon}) / B_{k}\right) \times\left\langle\varphi^{*}\right\rangle \cong \mathbb{Z} / 2^{k-1} \mathbb{Z} \times \mathbb{Z} / 2 \mathbb{Z}
$$

and $n_{k}=2$ as before. In the other case, $F_{k} / B_{k}$ is a cyclic extension of degree $2^{k}$ with quadratic subextension $B_{k}(\varepsilon)=B_{k}(\sqrt{\varepsilon})$. Any extension of $\varphi \in \operatorname{Gal}\left(B_{k}(\varepsilon) / B_{k}\right)$ to $F_{k}$ is then a generator of $\operatorname{Gal}\left(F_{k} / B_{k}\right)$, and we have $n_{k}=0$.

For $k=2$, any of the elements $\sqrt{2}$ and $\sqrt{\varepsilon} \pm 1 / \sqrt{\varepsilon}$ generates the quadratic extension $B_{2}(\sqrt{\varepsilon})=B_{2}(\sqrt{2})$ of $B_{2}$. The extension $B_{2} \subset B_{2}(\sqrt[4]{\varepsilon})=F_{2}$ is of degree 4 and has a quadratic subextension generated by $\sqrt{\varepsilon}=\sqrt{(a+1) / 2}+$ $\sqrt{(a-1) / 2}$. If $a+1$ is a square, then $\sqrt{\varepsilon}$ has norm -1 in $B_{k}$ and $F_{2} / B_{2}$ is a cyclic extension. If $a-1$ is a square, then $\sqrt{\varepsilon}$ has norm 1 in $B_{k}$ and $F_{2} / B_{2}$ is a $V_{4}$-extension. The corresponding values of $n_{2}$ are $n_{2}=0$ and $n_{2}=2$.

For $a+1$ a square we find $1 / 2-\delta_{K}^{-}=1 / 4+0+\sum_{k \geq 3} 2^{-k} \cdot 2 \cdot 2^{-k}=7 / 24$ and $\delta_{K}^{-}=5 / 24$. For $a-1$ a square we find a finite $\operatorname{sum} 1 / 2-\delta_{K}^{-}=1 / 4+1 / 8$ and $\delta_{K}^{-}=1 / 8$. This finishes the proof of the theorem.

\section{References}

[1] C. Ballot, Density of prime divisors of linear recurrences, Mem. Amer. Math. Soc. 551 (1995).

[2] H. Hasse, Über die Dichte der Primzahlen $p$, für die eine vorgegebene ganzrationale Zahl $a \neq 0$ von gerader bzw. ungerader Ordnung mod $p$ ist, Math. Ann. 166 (1966), $19-23$.

[3] J. C. Lagarias, The set of primes dividing the Lucas numbers has density 2/3, Pacific J. Math. 118 (1985), 449-461; Errata: ibid. 162 (1994), 393-397.

[4] P. Moree, On the prime density of Lucas sequences, J. Théor. Nombres Bordeaux 8 (1996), 449-459. 
[5] P. Riben boim, The New Book of Prime Number Records, Springer, New York, 1995.

[6] P. Stevenhagen, Prime densities for second order torsion sequences, preprint.

Max-Planck-Institut für Mathematik

Gottfried-Claren-Str. 26

53225 Bonn, Germany

E-mail: moree@mpim.bonn.mpg.de
Faculteit WINS

Universiteit van Amsterdam Plantage Muidergracht 24

1018 TV Amsterdam, The Netherlands

E-mail: psh@wins.uva.nl

Received on 7.4.1997

and in revised form on 24.7 .1997 